Теорія Ймовір. та Матем. Статист. Вип. 74, 2006
Theor. Probability and Math. Statist.

No. 74, 2007, Pages 163-170 S 0094-9000(07)00705-3

Article electronically published on July 5, 2007

\title{
ON THE ITERATED A POSTERIORI DISTRIBUTION IN BAYESIAN STATISTICS
}

UDC 519.21

\author{
F. RECKER
}

\begin{abstract}
In theoretical considerations a Bayesian experiment consisting of many independently drawn samples is usually modeled by a product space. However, in some applications, as e.g. pattern recognition, the mathematical model is different. This model will be presented and a rigid measure-theoretic proof will be given showing that both models deliver the same a posteriori distribution.
\end{abstract}

\section{INTRODUCTION}

In Bayesian statistics one has an a priori distribution on the parameter space which describes the knowledge about the true parameter before the outcome of the statistical experiment is known. After a realization has occurred, one gets the a posteriori distribution which can be used to define Bayesian tests or Bayesian estimators. Actually, the realization is often a vector $x=\left(x_{1}, \ldots, x_{n}\right)$ and all $x_{i}$ are assumed to be realizations of i.i.d. random variables. The adequate model is hence to use a product space. Among the huge amount of literature concerning Bayesian statistics, we mention 1, 2 for a general introduction and especially [3, 4, 5, for asymptotic results, i.e. results concerning the convergence of the a posteriori distribution if $n$ tends to infinity.

However this model has one shortcoming. When determining the a posteriori distribution, one has to go through some calculations. One especially has to do the calculation again and again if one increases $n$ and wishes to have intermediate a posteriori distributions. Therefore, in applications with this demand, such as pattern recognition (cf. [6]) or visualization of limit theorems in Bayesian statistics (cf. [7, pp. 175-180] and [8, pp. 1215]), a different approach is chosen. One starts with the above-described setting for one experiment. After the sample $x_{1}$ has occurred, the a priori distribution is replaced by the respective a posteriori distribution. The second sample $x_{2}$ yields again an a posteriori distribution which is taken as the new a priori distribution and so on. We call this concept the iterated a posteriori distribution. The natural question is of course: Having a realization $x=\left(x_{1}, \ldots, x_{n}\right)$, do both models deliver the same final distribution over the parameter space? A second question is: Is the ordering of the realizations important?

As far as we know, these questions have never been answered explicitly, but of course one assumes (or hopes?) that the answers will be yes in the first case and no in the second case. This can be verified with a short calculation for the discrete case. In this paper we will answer these questions for the general case. We will prove that under some measurability conditions both models deliver almost surely the same a posteriori distribution. Furthermore, we will prove that the a posteriori distribution does not depend

2000 Mathematics Subject Classification. Primary 62F15, $62 \mathrm{C} 12$.

Key words and phrases. Bayesian inference, a posteriori distribution.

(C)2007 American Mathematical Society 
on the ordering of the realizations. In particular even in a long running experiment the later realizations do not have a greater influence on the a posteriori distribution than the first realization.

In Section 2 we will introduce the necessary notation and state the theorem. The proof follows in Section 3 .

\section{Notation AND THE MAIN THEOREM}

A Bayesian experiment is a tuple $(\Gamma \times \mathbb{H}, \mathcal{G} \otimes \mathcal{H}, Q \otimes P)$ where $(\mathbb{H}, \mathcal{H})$ is a measurable space (the sample space) and $(\Gamma, \mathcal{G}, Q)$ is a Polish probability space (the parameter space). Here $Q$ is the a priori distribution and describes the knowledge about the unknown true parameter $\bar{\gamma} \in \Gamma$. Finally, $P$ is a stochastic kernel from $(\Gamma, \mathcal{G})$ to $(\mathbb{H}, \mathcal{H})$. In particular, $P(\gamma, \cdot)$ is a probability measure over $(\mathbb{H}, \mathcal{H})$ for all $\gamma \in \Gamma$, and $P(\bar{\gamma}, \cdot)$ is the true probability measure.

The a posteriori distribution is a stochastic kernel $H$ from $(\mathbb{H}, \mathcal{H})$ to $(\Gamma, \mathcal{G})$ which fulfills

$$
Q \otimes P=H \otimes(Q \otimes P)_{\pi_{\mathbb{H}}},
$$

where $(Q \otimes P)_{\pi_{\mathbb{H}}}$ is the marginal distribution of $Q \otimes P$ on $(\mathbb{H}, \mathcal{H})$. Since $(\Gamma, \mathcal{G})$ is Polish, $H$ exists (as a regular conditional distribution) and it is $(Q \otimes P)_{\pi_{\mathbb{H}}}$-a.s. determined. We write $f(Q \otimes P)^{\pi_{\Gamma} \mid \pi_{\mathbb{H}}}$ for the set of all these regular conditional distributions (cf. [2, Anhang A 2]). If $x \in \mathbb{H}$ is a realization, then $H(x, \cdot)$ is taken as the new distribution for the unknown true parameter $\bar{\gamma} \in \Gamma$. We remark that there is a slight inconsistency in the notation which is however usual in the literature: The term "a posteriori distribution" is used for the kernel $H$ and for the probability measure $H(x, \cdot)$.

If $\left(\Gamma \times \mathbb{H}_{i}, \mathcal{G} \otimes \mathcal{H}_{i}, Q \otimes P_{i}\right)$ are Bayesian experiments for $i=1,2$, then $(\Gamma \times \mathbb{H}, \mathcal{G} \otimes$ $\mathcal{H}, Q \otimes P)$ with $\mathbb{H}=\mathbb{H}_{1} \times \mathbb{H}_{2}, \mathcal{H}=\mathcal{H}_{1} \otimes \mathcal{H}_{2}$, and $P(\gamma, \cdot)=P_{1}(\gamma, \cdot) \otimes P_{2}(\gamma, \cdot)$ for all $\gamma \in \Gamma$ is also a Bayesian experiment. We will call it the product experiment of the experiments $\left(\Gamma \times \mathbb{H}_{i}, \mathcal{G} \otimes \mathcal{H}_{i}, Q \otimes P_{i}\right), i=1,2$. In the special case that both $\left(\Gamma \times \mathbb{H}_{i}, \mathcal{G} \otimes \mathcal{H}_{i}, Q \otimes P_{i}\right)$ are copies of the same $\left(\Gamma \times \mathbb{H}_{0}, \mathcal{G} \otimes \mathcal{H}_{0}, Q \otimes P_{0}\right)$, we will call $(\Gamma \times \mathbb{H}, \mathcal{G} \otimes \mathcal{H}, Q \otimes P)$ the second power of $\left(\Gamma \times \mathbb{H}_{0}, \mathcal{G} \otimes \mathcal{H}_{0}, Q \otimes P_{0}\right)$.

Let $\left(\Gamma \times \mathbb{H}_{0}, \mathcal{G} \otimes \mathcal{H}_{0}, Q \otimes P_{0}\right)$ be a Bayesian experiment with an a posteriori distribution $H_{1}$. If a sample $x \in \mathbb{H}_{0}$ occurs in the iterated model, then we get a new Bayesian experiment $\left(\Gamma \times \mathbb{H}_{0}, \mathcal{G} \otimes \mathcal{H}_{0}, H_{1}(x, \cdot) \otimes P_{0}\right)$. The a posteriori distribution for this experiment depends on $x$ and is an element of $f\left(H_{1}(x, \cdot) \otimes P_{0}\right)^{\pi_{\Gamma} \mid \pi_{\mathbb{H}_{0}}}$.

The questions raised in the introduction are answered for $n=2$ in the following theorem.

Theorem 2.1. Let $\left(\Gamma \times \mathbb{H}_{0}, \mathcal{G} \otimes \mathcal{H}_{0}, Q \otimes P_{0}\right)$ be a Bayesian experiment, and let

$$
(\Gamma \times \mathbb{H}, \mathcal{G} \otimes \mathcal{H}, Q \otimes P)
$$

be the second power of $\left(\Gamma \times \mathbb{H}_{0}, \mathcal{G} \otimes \mathcal{H}_{0}, Q \otimes P_{0}\right)$. Furthermore let $H \in f(Q \otimes P)^{\pi_{\Gamma} \mid \pi_{\mathbb{H}}}$, $H_{1} \in f\left(Q \otimes P_{0}\right)^{\pi_{\Gamma} \mid \pi_{\mathbb{H}_{0}}}$, and let $H_{2}^{x} \in f\left(H_{1}(x, \cdot) \otimes P_{0}\right)^{\pi_{\Gamma} \mid \pi_{\mathbb{H}_{0}}}$ for all $x \in \mathbb{H}_{0}$ be such that the mapping

$$
(x, y) \mapsto H_{2}^{x}(y, G)
$$

is $\mathcal{H}$-measurable for all $G \in \mathcal{G}$. Then there exists a $(Q \otimes P)_{\pi_{\mathbb{H}}}$-null-set $N$ such that

$$
H_{2}^{x}(y, G)=H((x, y), G)=H((y, x), G)=H_{2}^{y}(x, G), \quad(x, y) \in N^{c}, G \in \mathcal{G} .
$$

We remark that a similar statement holds for $n>2$. We will give a sketch of the proof of this generalization in the next section. 


\section{THE PROOF}

In this section we will prove Theorem 2.1 To this end, we have to show the claimed existence of the null-set $N$. It is obvious that, if $N$ exists, then it could be chosen with the additional property: If $(x, y) \in N$, then $(y, x) \in N$. To formalize this, we give the following definition: A set $M \subset \mathbb{H}_{0} \times \mathbb{H}_{0}$ is symmetric if and only if for all $x, y \in \mathbb{H}_{0}$ we have $(x, y) \in M \Longleftrightarrow(y, x) \in M$. The following short lemma shows that we can always assume w.l.o.g. that a null-set is symmetric.

Lemma 3.1. With the notation of Theorem 2.1 let $N$ be $a(Q \otimes P)_{\pi_{H}}$-null-set. Then there is a symmetric $(Q \otimes P)_{\pi_{\mathbb{H}}}$ null-set $\bar{N}$ with $N \subseteq \bar{N}$.

Proof. The mapping $Y: \mathbb{H}_{0} \times \mathbb{H}_{0} \rightarrow \mathbb{H}_{0} \times \mathbb{H}_{0}$ defined by $Y(x, y)=(y, x)$ is measurable and we have

$$
(Q \otimes P)_{\pi_{\mathbb{H}}}=\left((Q \otimes P)_{\pi_{\mathbb{H}}}\right)_{Y}
$$

Define

$$
\bar{N}:=N \cup\{(x, y) \mid(y, x) \in N\}=N \cup Y^{-1}(N) .
$$

Then $\bar{N}$ is measurable with measure zero, and $N \subseteq \bar{N}$.

The proof of Theorem 2.1 consists of two key lemmas. The first lemma shows that the a posteriori distribution for the product space model is almost surely symmetric.

Lemma 3.2. Let $\left(\Gamma \times \mathbb{H}_{0}, \mathcal{G} \otimes \mathcal{H}_{0}, Q \otimes P_{0}\right)$ be a Bayesian experiment and let

$$
(\Gamma \times \mathbb{H}, \mathcal{G} \otimes \mathcal{H}, Q \otimes P)
$$

be the second power. Then for each a posteriori distribution $H \in f(Q \otimes P)^{\pi_{\Gamma} \mid \pi_{\sharp}}$ there is a symmetric $(Q \otimes P)_{\pi_{\mathbb{H}}}$-null-set $N$ such that

$$
H((x, y), G)=H((y, x), G), \quad(x, y) \in N^{c}, G \in \mathcal{G} .
$$

This result could also be stated in the following way: The symmetric $\sigma$-algebra (i.e. the $\sigma$-algebra which consists of all symmetric sets of $\mathcal{H}$ ) is sufficient (cf. [9, chapter 2] for a definition of Bayesian sufficiency).

The second lemma is the core of the proof of Theorem 2.1. It states that the iterated a posteriori distribution is almost surely equal to the usual one. Note that we formulate this lemma for possibly different Bayesian experiments. The lemma can therefore be used to prove a generalization of Theorem 2.1 for $n$ samples by induction.

Lemma 3.3. Let $\left(\Gamma \times \mathbb{H}_{i}, \mathcal{G} \otimes \mathcal{H}_{i}, Q \otimes P_{i}\right), i=1,2$, be Bayesian experiments, let

$$
(\Gamma \times \mathbb{H}, \mathcal{G} \otimes \mathcal{H}, Q \otimes P)
$$

be the product experiment, let $H \in f(Q \otimes P)^{\pi_{\Gamma} \mid \pi_{\mathbb{H}}}, H_{1} \in f\left(Q \otimes P_{1}\right)^{\pi_{\Gamma} \mid \pi_{\mathbb{H}_{1}}}$ and let

$$
H_{2}^{x} \in f\left(H_{1}(x, \cdot) \otimes P_{2}\right)^{\pi_{\Gamma} \mid \pi_{\mathbb{H}_{2}}}, \quad x \in \mathbb{H}_{1},
$$

be such that the mapping

$$
(x, y) \mapsto H_{2}^{x}(y, G)
$$

is $\mathcal{H}$-measurable for all $G \in \mathcal{G}$. Then there exists a $(Q \otimes P)_{\pi_{\mathbb{H}}}$-null-set $N$ such that

$$
H_{2}^{x}(y, G)=H((x, y), G), \quad(x, y) \in N^{c}, G \in \mathcal{G} .
$$

Suppose now for a moment that the above lemmas were proved. Then we can prove Theorem 2.1 .

Proof of Theorem 2.1. We choose the null-set $N$ as the union of the exception sets that appear in Lemma 3.2 and Lemma 3.3. W.l.o.g. we can assume that $N$ is symmetric (cf. Lemma 3.1). Then for all $(x, y) \in N^{c}$ and $G \in \mathcal{G}$ we have

$$
H_{2}^{x}(y, G)=H((x, y), G)=H((y, x), G)=H_{2}^{y}(x, G) .
$$


It remains to prove Lemma 3.2 and Lemma 3.3 . This will be done in the rest of this section. First we will derive some useful equations for the product space. With the notation of Section 2 (i.e. $\mathbb{H}=\mathbb{H}_{1} \times \mathbb{H}_{2}, \mathcal{H}=\mathcal{H}_{1} \otimes \mathcal{H}_{2}$ and $P(\gamma, \cdot)=P_{1}(\gamma, \cdot) \otimes P_{2}(\gamma, \cdot)$ for all $\gamma \in \Gamma$ ) we have

$$
P\left(\gamma, A_{1} \times A_{2}\right)=P_{1}\left(\gamma, A_{1}\right) \cdot P_{2}\left(\gamma, A_{2}\right), \quad A_{1} \in \mathcal{H}_{1}, A_{2} \in \mathcal{H}_{2}, \gamma \in \Gamma,
$$

respectively,

$$
P(\gamma, A)=\int_{\mathbb{H}_{1}} P_{2}\left(\gamma, A_{x}\right) P_{1}(\gamma, d x)=\int_{\mathbb{H}_{2}} P_{1}\left(\gamma, A_{y}\right) P_{2}(\gamma, d y), \quad A \in \mathcal{H}, \gamma \in \Gamma,
$$

where $A_{x}$, resp. $A_{y}$, is the $x$-cut, resp. $y$-cut of $A$. Moreover, the measure $Q \otimes P$ fulfills

$$
\begin{aligned}
(Q \otimes & P)\left(G \times A_{1} \times A_{2}\right) \\
& =\int_{G} P\left(\gamma, A_{1} \times A_{2}\right) Q(d \gamma) \\
& =\int_{G} P_{1}\left(\gamma, A_{1}\right) \cdot P_{2}\left(\gamma, A_{2}\right) Q(d \gamma), \quad A_{1} \in \mathcal{H}_{1}, A_{2} \in \mathcal{H}_{2}, G \in \mathcal{G},
\end{aligned}
$$

and

$$
\begin{aligned}
(Q \otimes & P)(G \times A) \\
& =\int_{G} P(\gamma, A) Q(d \gamma) \\
& =\int_{G}\left(\int_{\mathbb{H}_{1}} P_{2}\left(\gamma, A_{x}\right) P_{1}(\gamma, d x)\right) Q(d \gamma), \quad A \in \mathcal{H}, G \in \mathcal{G} .
\end{aligned}
$$

Next we define a special a posteriori distribution which is symmetric without any exceptions.

Lemma 3.4. Let $\left(\Gamma \times \mathbb{H}_{0}, \mathcal{G} \otimes \mathcal{H}_{0}, Q \otimes P_{0}\right)$ be a Bayesian experiment and let

$$
(\Gamma \times \mathbb{H}, \mathcal{G} \otimes \mathcal{H}, Q \otimes P)
$$

be the second power. Then there is an a posteriori distribution $\bar{H} \in f(Q \otimes P)^{\pi_{\Gamma} \mid \pi_{\mathbb{H}}}$ such that for all $(x, y) \in \mathbb{H}$ and all $G \in \mathcal{G}$ we have

$$
\bar{H}((x, y), G)=\bar{H}((y, x), G) .
$$

Proof. We want to distinguish the two instances of $\left(\Gamma \times \mathbb{H}_{0}, \mathcal{G} \otimes \mathcal{H}_{0}, Q \otimes P_{0}\right)$. Therefore, let $\left(\Gamma \times \mathbb{H}_{i}, \mathcal{G} \otimes \mathcal{H}_{i}, Q \otimes P_{i}\right)=\left(\Gamma \times \mathbb{H}_{0}, \mathcal{G} \otimes \mathcal{H}_{0}, Q \otimes P_{0}\right), i=1,2$. Then $(\Gamma \times \mathbb{H}, \mathcal{G} \otimes \mathcal{H}, Q \otimes P)$ is the product experiment of these two experiments. In particular $\mathbb{H}=\mathbb{H}_{0} \times \mathbb{H}_{0}=\mathbb{H}_{1} \times \mathbb{H}_{2}$. Let $Y: \mathbb{H}_{1} \times \mathbb{H}_{2} \rightarrow \mathbb{H}_{1} \times \mathbb{H}_{2}$ be defined as $Y(x, y)=(y, x)$. For all $A_{1} \in \mathcal{H}_{1}$ and $A_{2} \in \mathcal{H}_{2}$ it follows from $\mathcal{H}_{1}=\mathcal{H}_{2}$ that

$$
Y^{-1}\left(A_{1} \times A_{2}\right)=A_{2} \times A_{1} \in \mathcal{H}_{2} \otimes \mathcal{H}_{1}=\mathcal{H}_{1} \otimes \mathcal{H}_{2} .
$$

The set of all $A_{1} \times A_{2} \in \mathcal{H}_{1} \times \mathcal{H}_{2}$ is a generating system for $\mathcal{H}_{1} \otimes \mathcal{H}_{2}=\mathcal{H}$. Hence $Y$ is $\mathcal{H}$ - $\mathcal{H}$-measurable. Again let $A_{1} \in \mathcal{H}_{1}$ and $A_{2} \in \mathcal{H}_{2}$. Then

$$
\begin{aligned}
& \left((Q \otimes P)_{\pi_{\mathbb{H}}}\right)_{Y}\left(A_{1} \times A_{2}\right)=(Q \otimes P)_{\pi_{\mathbb{H}}}\left(Y^{-1}\left(A_{1} \times A_{2}\right)\right) \\
& \underset{(3.6)}{=}(Q \otimes P)_{\pi_{H}}\left(A_{2} \times A_{1}\right) \\
& =(Q \otimes P)\left(\Gamma \times A_{2} \times A_{1}\right) \\
& \underset{\substack{3.3 \\
P_{1}=P_{2}}}{=}(Q \otimes P)\left(\Gamma \times A_{1} \times A_{2}\right) \\
& =(Q \otimes P)_{\pi_{\mathbb{H}}}\left(A_{1} \times A_{2}\right) .
\end{aligned}
$$


Hence (3.7) and the measure-extension theorem show that

$$
\left((Q \otimes P)_{\pi_{\mathbb{H}}}\right)_{Y}=(Q \otimes P)_{\pi_{\mathbb{H}}} .
$$

Since $(\Gamma, \mathcal{G})$ is Polish, there exists a regular conditional distribution

$$
H \in f(Q \otimes P)^{\pi_{\Gamma} \mid \pi_{\mathbb{H}}} .
$$

Here $H$ is a stochastic kernel from $(\mathbb{H}, \mathcal{H})$ to $(\Gamma, \mathcal{G})$. We define

$$
\bar{H}((x, y), G):=\frac{1}{2}(H((x, y), G)+H(Y(x, y), G)) .
$$

It can be shown easily that $\bar{H}$ is a stochastic kernel from $(\mathbb{H}, \mathcal{H})$ to $(\Gamma, \mathcal{G})$. For all $A \in \mathcal{H}$ and $G \in \mathcal{G}$ it follows that

$$
\left(\bar{H} \otimes(Q \otimes P)_{\pi_{\mathbb{H}}}\right)(G \times A) \underset{[3.8}{=}\left(H \otimes(Q \otimes P)_{\pi_{\mathbb{H}}}\right)(G \times A) \underset{(2.1)}{=}(Q \otimes P)(G \times A) .
$$

Using the measure-extension theorem, we conclude that

$$
Q \otimes P=\bar{H} \otimes(Q \otimes P)_{\pi_{\mathbb{H}}} .
$$

Therefore $\bar{H} \in f(Q \otimes P)^{\pi_{\Gamma} \mid \pi_{\mathbb{H}}}$. Furthermore $\bar{H}$ fulfills (3.5) by construction.

Proof of Lemma 3.2. Choose $\bar{H}$ according to Lemma 3.4. Since $(\Gamma, \mathcal{G})$ is Polish, the a posteriori distribution $H$ is $(Q \otimes P)_{\pi_{\mathbb{H}}}$-a.s. determined, i.e. there is a $(Q \otimes P)_{\pi_{\mathbb{H}}}$-nullset $N$, such that

$$
H((x, y), G)=\bar{H}((x, y), G), \quad(x, y) \in N^{c}, G \in \mathcal{G} .
$$

Due to Lemma 3.1 we can assume w.l.o.g. that $N$ is symmetric, which proves the claim.

The key idea in the proof of Lemma 3.3 is this: Express the marginal distribution on the sample space with the help of a stochastic kernel. The existence of this kernel however is a little surprising since we did not suppose that the sample spaces are Polish.

Lemma 3.5. Let $\left(\Gamma \times \mathbb{H}_{i}, \mathcal{G} \otimes \mathcal{H}_{i}, Q \otimes P_{i}\right), i=1,2$, be Bayesian experiments, let

$$
(\Gamma \times \mathbb{H}, \mathcal{G} \otimes \mathcal{H}, Q \otimes P)
$$

be the product experiment, and let $H_{1} \in f\left(Q \otimes P_{1}\right)^{\pi_{\Gamma} \mid \pi_{\mathbb{H}_{1}}}$ (such an $H_{1}$ always exists). Define the mapping $K: \mathbb{H}_{1} \times \mathcal{H}_{2} \rightarrow \mathbb{R}$ as

$$
K\left(x, A_{2}\right):=\left(H_{1}(x, \cdot) \otimes P_{2}\right)_{\pi_{\mathbb{H}_{2}}}\left(A_{2}\right) .
$$

Then $K$ is a stochastic kernel from $\left(\mathbb{H}_{1}, \mathcal{H}_{1}\right)$ to $\left(\mathbb{H}_{2}, \mathcal{H}_{2}\right)$ and

$$
(Q \otimes P)_{\pi_{\mathbb{H}}}=\left(Q \otimes P_{1}\right)_{\pi_{\mathbb{H}_{1}}} \otimes K
$$

(i.e. $\left.K \in f\left((Q \otimes P)_{\pi_{\mathbb{H}}}\right)^{\pi_{\mathbb{H}_{2}} \mid \pi_{\mathbb{H}_{1}}}\right)_{\text {. }}$.

Proof. Since $(\Gamma, \mathcal{G})$ is Polish, $H_{1} \in f\left(Q \otimes P_{1}\right)^{\pi_{\Gamma} \mid \pi_{\mathbb{H}_{1}}}$ and $H \in f(Q \otimes P)^{\pi_{\Gamma} \mid \pi_{\mathbb{H}}}$ exist. For all $x \in \mathbb{H}_{1}$

$$
K(x, \cdot)=\left(H_{1}(x, \cdot) \otimes P_{2}\right)_{\pi_{\mathbb{H}_{2}}}
$$

is a probability measure over $\left(\mathbb{H}_{2}, \mathcal{H}_{2}\right)$. For the a posteriori distribution $H_{1}$ follows as in (2.1)

$$
Q \otimes P_{1}=H_{1} \otimes\left(Q \otimes P_{1}\right)_{\pi_{\mathbb{H}_{1}}}
$$


Let $A_{2} \in \mathcal{H}_{2}$ be arbitrary. We have for all $x \in \mathbb{H}_{1}$ that

$$
\begin{aligned}
K\left(x, A_{2}\right) & =\left(H_{1}(x, \cdot) \otimes P_{2}\right)_{\pi_{\mathbb{H}_{2}}}\left(A_{2}\right)=\left(H_{1}(x, \cdot) \otimes P_{2}\right)\left(\Gamma \times A_{2}\right) \\
& =\int_{\Gamma} P_{2}\left(\gamma, A_{2}\right) H_{1}(x, d \gamma) .
\end{aligned}
$$

The mapping

$$
(x, \gamma) \mapsto P_{2}\left(\gamma, A_{2}\right)
$$

is $\mathcal{H}_{1} \otimes \mathcal{G}$-measurable (since it does not depend on $x$ and $P_{2}\left(\cdot, A_{2}\right)$ is $\mathcal{G}$-measurable) and $H_{1}$ is a stochastic kernel from $\left(\mathbb{H}_{1}, \mathcal{H}_{1}\right)$ to $(\Gamma, \mathcal{G})$. From Fubini's theorem for stochastic kernels it follows that the mapping

$$
x \mapsto \int_{\Gamma} P_{2}\left(\gamma, A_{2}\right) H_{1}(x, d \gamma)
$$

is $\mathcal{H}_{1}$-measurable. Therefore (3.12) shows that

$$
x \mapsto K\left(x, A_{2}\right)
$$

is $\mathcal{H}_{1}$-measurable. Hence we have shown that $K$ is a stochastic kernel.

Let $A_{1} \in \mathcal{H}_{1}$ and $A_{2} \in \mathcal{H}_{2}$. Then

$$
\begin{aligned}
& (Q \otimes P)_{\pi_{\mathbb{H}}}\left(A_{1} \times A_{2}\right)=(Q \otimes P)\left(\Gamma \times A_{1} \times A_{2}\right) \\
& \quad=\int_{\Gamma} P_{1}\left(\gamma, A_{1}\right) P_{2}\left(\gamma, A_{2}\right) Q(d \gamma) \\
& \quad=\int_{\Gamma}\left(\int_{A_{1}} P_{2}\left(\gamma, A_{2}\right) P_{1}(\gamma, d x)\right) Q(d \gamma) \\
& \underset{\text { Fubini }}{=} \int_{\Gamma \times A_{1}} P_{2}\left(\gamma, A_{2}\right)\left(Q \otimes P_{1}\right)(d(x, \gamma)) \\
& \underset{\quad=}{=} \int_{\Gamma \times A_{1}} P_{2}\left(\gamma, A_{2}\right)\left(H_{1} \otimes\left(Q \otimes P_{1}\right)_{\pi_{\mathbb{H}_{1}}}\right)(d(x, \gamma)) \\
& \quad=\int_{A_{1}}\left(\int_{\Gamma} P_{2}\left(\gamma, A_{2}\right) H_{1}(x, d \gamma)\right)\left(Q \otimes P_{1}\right)_{\pi_{\mathbb{H}_{1}}}(d x) \\
& =\int_{A_{1}} K\left(x, A_{2}\right)\left(Q \otimes P_{1}\right)_{\pi_{\mathbb{H}_{1}}}(d x)=\left(\left(Q \otimes P_{1}\right)_{\pi_{\mathbb{H}_{1}}} \otimes K\right)\left(A_{1} \times A_{2}\right) .
\end{aligned}
$$

In view of the measure-extension theorem, equation (3.10) is valid.

Proof of Lemma 3.3. Let $A \in \mathcal{H}$ and $G \in \mathcal{G}$ be arbitrary. We choose $K$ as in Lemma 3.5. As in (2.1) it follows for $H_{2}^{x}$ that

$$
H_{2}^{x} \otimes\left(H_{1}(x, \cdot) \otimes P_{2}\right)_{\pi_{\mathbb{H}_{2}}}=H_{1}(x, \cdot) \otimes P_{2}, \quad x \in \mathbb{H}_{1} .
$$

Using (3.9), we get

$$
H_{2}^{x} \otimes K(x, \cdot)=H_{1}(x, \cdot) \otimes P_{2}, \quad x \in \mathbb{H}_{1} .
$$


The required measurability yields for all $A \in \mathcal{H}$

$$
\begin{aligned}
& \int_{A} H_{2}^{x}(y, G)(Q \otimes P)_{\pi_{H}}(d(x, y)) \\
& \underset{\text { (3.10) }}{=} \int_{A} H_{2}^{x}(y, G)\left(\left(Q \otimes P_{1}\right)_{\pi_{\mathbb{H}_{1}}} \otimes K\right)(d(x, y)) \\
& \underset{\text { Fubini }}{=} \int_{\mathbb{H}_{1}}\left(\int_{A_{x}} H_{2}^{x}(y, G) K(x, d y)\right)\left(Q \otimes P_{1}\right)_{\pi_{\mathbb{H}_{1}}}(d x) \\
& =\int_{\mathbb{H}_{1}}\left(H_{2}^{x} \otimes K(x, \cdot)\right)\left(G \times A_{x}\right)\left(Q \otimes P_{1}\right)_{\pi_{\mathbb{H}_{1}}}(d x) \\
& \underset{\text { (3.13) }}{=} \int_{\mathbb{H}_{1}}\left(H_{1}(x, \cdot) \otimes P_{2}\right)\left(G \times A_{x}\right)\left(Q \otimes P_{1}\right)_{\pi_{\mathbb{H}_{1}}}(d x) \\
& =\int_{\mathbb{H}_{1}}\left(\int_{G} P_{2}\left(\gamma, A_{x}\right) H_{1}(x, d \gamma)\right)\left(Q \otimes P_{1}\right)_{\pi_{\mathbb{H}_{1}}}(d x) \\
& \underset{\text { Fubini }}{=} \int_{G \times \mathbb{H}_{1}} P_{2}\left(\gamma, A_{x}\right)\left(\left(Q \otimes P_{1}\right)_{\pi_{\mathbb{H}_{1}}} \otimes H_{1}\right)(d(x, \gamma)) \\
& \underset{H_{1} \in f\left(Q \otimes P_{1}\right)^{\pi_{\Gamma} \mid \pi_{\mathbb{H}_{1}}}}{=} \int_{G \times \mathbb{H}_{1}} P_{2}\left(\gamma, A_{x}\right)\left(Q \otimes P_{1}\right)(d(x, \gamma)) \\
& \text { Fubini } \int_{G}\left(\int_{\mathbb{H}_{1}} P_{2}\left(\gamma, A_{x}\right) P_{1}(\gamma, d x)\right) Q(d \gamma) \\
& \underset{(3.4)}{=}(Q \otimes P)(G \times A)
\end{aligned}
$$

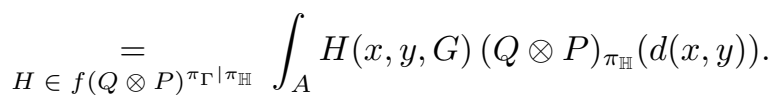

Since $A \in \mathcal{H}$ was arbitrary, we have $H_{2}^{x}(y, G)=H(x, y, G)(Q \otimes P)_{\pi_{\mathbb{H}}}$-a.s. Hence there exists a $(Q \otimes P)_{\pi_{\mathbb{H}}}$-null-set $N_{G}$ depending on $G$, with

$$
H_{2}^{x}(y, G)=H((x, y), G), \quad(x, y) \in N_{G}^{c} .
$$

We can now apply a standard technique: Since $(\Gamma, \mathcal{G})$ is Polish, $\mathcal{G}$ has a countable ring $\mathcal{R}$ as a generating system. For the $(Q \otimes P)_{\pi_{\mathbb{H}}}$-null-set $N:=\bigcup_{G \in \mathcal{R}} N_{G}$ it follows that

$$
H_{2}^{x}(y, G)=H((x, y), G), \quad(x, y) \in N^{c}, G \in \mathcal{R} .
$$

From the measure-extension theorem it follows therefore that

$$
H_{2}^{x}(y, G)=H((x, y), G), \quad(x, y) \in N^{c}, G \in \mathcal{G} .
$$

\section{BIBLIOGRAPHY}

1. J. A. Hartigan, Bayes Theory, Springer, New York, 1983. MR715782 (85k:60008)

2. W. Eberl and O. Moeschlin, Mathematische Statistik, de Gruyter, Berlin, 1982. MR670752 (84e:62003)

3. L. LeCam, Asymptotic Methods in Statistical Decision Theory, Springer, New York, 1986. MR 856411 (88a:62004)

4. L. LeCam, Asymptotics in Statistics, Springer, New York, 1990. MR1066869 (92k:62050)

5. P. Rao, Asymptotic Theory of Statistical Inference, John Wiley, New York, 1987. MR 874342 (88b:62001)

6. T. Y. Young and T. W. Calvert, Classification, Estimation, and Pattern Recognition, Elsevier Science Publishers, Amsterdam, 1974. MR0350975 (50:3467)

7. O. Moeschlin, E. Grycko, C. Pohl, and F. Steinert, Experimental Stochastics, Springer, BerlinNew York, 1998. 
8. O. Moeschlin and F. Steinert, Bayessche Statistik, Birkhäuser, Basel, 1995.

9. J. P. Florens, M. Mouchart, and J. M. Rolin, Elements of Bayesian Statistics, Marcel Dekker, New York, 1990. MR,1051656 (91g:62004)

Department of Mathematics, University of Hagen, D-58084 Hagen, Germany

E-mail address: Frank.Recker@FernUni-Hagen.de 